Results Adolescents who were more physically active (total PA or minutes of MVPA) had a reduced odds of depression (adjusted OR per $100 \mathrm{cpm}$ total PA: 0.92 (95\% CI 0.88 to 0.97 ); adjusted OR per 15 min MVPA: 0.94 (95\% CI 0.88 to 1.01)). In a multivariable model including both total PA and the percentage of time spent in MVPA, MVPA was not independently associated with depression (adjusted OR MVPA (tertiles) medium 1.06 (95\% CI 0.88 to 1.28), high 0.99 (95\% CI 0.82 to 1.20$)$ ).

Conclusion The total amount of PA undertaken was associated with adolescent depression but the amount of time spent in MVPA, once total PA was accounted for, was not. Thus, the relevance of the intensity of the activity may be different for different dimensions of health. This would have important implications for public health messages if confirmed in longitudinal studies and randomised controlled trials.

\section{P2-328 EFFECTS OF NEIGHBOURHOOD-LEVEL PREDICTORS ON BODY MASS INDEX (BMI) TRAJECTORIES AMONG YOUNG CHILDREN IN CANADA}

doi:10.1136/jech.2011.142976k.60

Dr Piotr Wilk. ${ }^{*}$ University of Western Ontario, London, Ontario

Introduction Childhood obesity is a major public health concern in Canada as nearly $17 \%$ of children between 2 and 11 are overweight and more than $7 \%$ are obese. The objective of this study is to examine whether neighbourhood-level predictors affect BMI trajectories among young children.

Methods We conducted a secondary data analysis of the National Longitudinal Survey of Children and Youth. A cohort of over 6000 2- and 3-year-old children were followed between 1994 and 2004 in the sequence of bi-annual interviews. Multi-cohort latent growth curve modelling techniques for hierarchical data were employed to assess an independent effect of neighbourhood characteristics on BMI trajectories, after controlling for a number of child- and familylevel covariates. Neighbourhood conditions were assessed by indicators related to the physical environment (built and physical) in which the child lives, as well as factors related to socio-economic status of its inhabitants.

Results Overall, between the ages of 2 and 12, the estimated BMI trajectory followed the expected U-shaped pattern. The parameter estimates of this trajectory varied significantly, both across-children and across-neighbourhoods. In the unadjusted model, the betweenneighbourhood variance constituted approximately $20 \%$ of the total variance in these estimates. The results from the final model suggest that a statistically significant portion of the between-neighbourhood variance was accounted by the proposed neighbourhood-level predictors.

Conclusion Neighbourhood-level predictors were identified as significant predictors of the variance in BMI trajectories, suggesting that the neighbourhood characteristics play an important role in shaping BMI trajectories among young children in Canada.

\section{P2-329 WHAT MEDICAL CONDITIONS AND MEDICATIONS USED TO TREAT THE MEDICAL CONDITIONS INCREASE THE RISK OF SUBSEOUENT MOTOR VEHICLE INJURIES: RESULTS OF THE CANADIAN NATIONAL POPULATION HEALTH SURVEY}

doi:10.1136/jech.2011.142976k.61

P Wilk, ${ }^{*}$ E Vingilis. University of Western Ontario, London, Ontario, Canada

Introduction The last 2 decades have seen an increased interest in the relationships between medical conditions and medication use and motor vehicle injuries (MVIs). The objective of this study is to examine the effects of various medical conditions and medications used to treat the conditions on subsequent MVIs.

Method The National Population Health Survey is a large nationally representative sample of Canadians who have been surveyed every 2 years since 1994. Self-reported medical conditions and medication use were examined in relation to MVIs reported in the subsequent wave of the survey. Respondents were queried on whether they had any of the following long-term conditions: asthma, arthritis/rheumatism, back problems, high blood pressure, migraine headaches, pain, diabetes and heart disease; measures of distress and depression were also included. They were also asked whether they had taken medications to treat these conditions. Medical conditions and medications were subjected to regression analyses where medical conditions and medications served as controls for each other.

Results The results found that asthma, back problems, migraine and distress showed statistically significant increased risk of subsequent MVIs. Various medications (asthma medication, Demerol, codeine, pain medication and, sleeping medication) were also associated with increased risk of subsequent MVIs. Finally, for some medical conditions, medications have a protective effect while for other conditions, medications have independent effects on the risk of subsequent MVIs.

Conclusion This study suggests that the relationship between medical conditions and medications is complex and in need of further study.

\section{P2-330 ASSOCIATIONS OF 25-HYDROXYVITAMIN D2 AND D3 WITH CARDIOVASCULAR RISK FACTORS IN CHILDHOOD: A CROSS-SECTIONAL ANALYSIS IN THE AVON LONGITUDINAL STUDY OF PARENTS AND CHILDREN (ALSPAC)}

doi:10.1136/jech.2011.142976k.62

${ }^{1} \mathrm{D}$ Williams, ${ }^{* 1} \mathrm{~A}$ Fraser, ${ }^{2} \mathrm{~W}$ Fraser, ${ }^{3} \mathrm{~N}$ Sattar, ${ }^{4} \mathrm{~A}$ Hingorani, ${ }^{5} \mathrm{~J}$ Deanfield, ${ }^{1} \mathrm{G} D$ Smith, ${ }^{1} \mathrm{D}$ Lawlor. ${ }^{1}$ University of Bristol, Bristol, UK; ${ }^{2}$ University of Liverpool, Liverpool, UK; ${ }^{3}$ University of Glasgow, Glasgow, UK; ${ }^{4} \mathrm{UCL}$, London, UK; ${ }^{5} \mathrm{CH}$, London, UK

Introduction Some observational studies have associated low vitamin $\mathrm{D}$ status with increased cardiovascular disease (CVD) and risk factors in adults, but results from randomised controlled trials suggest these associations may not be causal. Trials have largely used supplementation with vitamin D2 and the availability of D3 supplements has led to the suggestion that this is more potent and null effects in previous trials may be because of inadequate dosage of vitamin $\mathrm{D}$.

Methods We conducted a cross-sectional study of 4274 children from the Avon Longitudinal Study of Parents and Children (ALSPAC), comparing associations of serum 25-hydroxyvitamin D2 $(25(\mathrm{OH}) \mathrm{D} 2)$ and 25-hydroxyvitamin D3 $(25(\mathrm{OH}) \mathrm{D} 3$ with several CVD risk factors: systolic and diastolic blood pressure (SBP; DBP), lipids (triglycerides, LDL-c, HDL-c, Apo-A1 and Apo-B) adiponectin, leptin, CRP and IL6.

Results In fully adjusted models including age, sex, ethnicity, socioeconomic position, waist circumference and mutual adjustment, $25(\mathrm{OH}) 3$ was positively associated with HDL-c (change per doubling of 25(OH)D3: $0.02 \mathrm{mmol} / \mathrm{l}$; $95 \%$ CI 0.0 to 0.04$)$ and ApoA1 $(2.7 \mathrm{mg} / \mathrm{dl} ; 1.5,3.8)$, and inversely associated with IL6 $(-7.8 \%$; $-12.3,-3.1)$. Equivalent analyses for $25(\mathrm{OH}) \mathrm{D} 2$ found positive associations with CRP $(8.0 \% ; 3.2,13.0)$ and IL6 (5.0\%; 1.5, 8.7). Neither exposure was associated with any other outcome. There was statistical evidence that associations of D2 and D3 differed for triglycerides, Apo-A1, adiponectin, CRP and IL6 (all $p$ values for heterogeneity $<0.04)$.

Conclusions $25(\mathrm{OH}) \mathrm{D} 2$ and $25(\mathrm{OH}) \mathrm{D} 3$ differ in their associations with CVD risk factors, but with no clear evidence in children that D3 is necessarily a more potent risk factor for CVD risk. 